\title{
ECONOMÍA COLABORATIVA: ACTUALIDAD EN EL SECTOR HOTELERO Y GREMIO DE LOS TAXISTAS DE LA CIUDAD DE PEREIRA
}

Carlos David Quinayas*, Mauricio Vargas Gutiérrez ${ }^{* *}$, Mauricio Zapata***

\section{Resumen}

La economía colaborativa es una tendencia que ha ido en crecimiento a nivel global y Colombia no es ajena a ella; hoy en día, las plataformas digitales hacen que el comercio sea cada vez más ágil y muchas personas lo aprueban y algunas no. El presente trabajo hace una investigación acerca de la percepción actual que tiene el gremio de los taxistas y el sector hotelero frente a las plataformas que forman parte de la economía colaborativa en transporte y servicio hotelero de la ciudad de Pereira. Entonces, con base en el planteamiento del problema encontrado en el sector tradicional de transporte y servicio hotelero, esta investigación sustenta la siguiente pregunta con relación a indagar sobre ¿cuál es la actualidad de la economía colaborativa en el sector hotelero y el gremio de los taxistas en la ciudad de Pereira?

* Estudiante, Administración de Negocios Internacionales. Fundación Universitaria del Área Andina. Correo: cquinayas2@estudiantes.areandina.edu.co

* Estudiante, Administración de Negocios Internacionales. Fundación Universitaria del Área Andina. Correo: mvargas45@estudiantes.areandina.edu.co

*** Estudiante, Administración de Negocios Internacionales. Fundación Universitaria del Área Andina. Correo: mzapata28@estudiantes.areandina.edu.co 


\section{Introducción}

Con la evolución de la tecnología, cada vez se facilitan más las cosas, y las tendencias a usar plataformas para adquirir bienes y servicios crece cada día más, lo que hace que el comercio crezca y las personas no tengan excusas a la hora de adquirir un servicio. La economía colaborativa sigue evolucionando a gran escala y hoy se puede evidenciar numerosas plataformas con tal fin. En el presente trabajo se pretende hacer énfasis en las plataformas más comunes del sector hotelero (Airbnb) y el servicio de transporte (Uber) en la ciudad de Pereira, donde estas se han visto atacadas por los gremios "legales" quienes intentan arduamente en eliminar del mercado a los usuarios que prestan servicios en estos aplicativos.

Por esta razón, se da a la tarea de realizar una investigación donde se aborden los diferentes conceptos que tiene el gremio de taxistas y el sector hotelero de la ciudad de Pereira acerca de las plataformas digitales, que permita saber los "perjuicios" que le dan a la economía tradicional, lo cual ha hecho que en ocasiones se revelen ante el Gobierno y soliciten la salida de estas plataformas del país.

En el presente trabajo se pretende abordar, en primer lugar, partiendo de antecedentes e información secundaria, para darle soporte a posibles hipótesis, para posteriormente adquirir información primaria, realizando un trabajo de campo con encuestas que constan de 13 preguntas, donde se cuestionarán los puntos principales a abordar en la investigación. Las limitaciones al trabajo son mínimas, pues se cuenta con bases de datos de la Cámara de Comercio de Pereira donde se encuentran los registros de todos los hoteles de la ciudad y, de igual manera, se cuenta con la base de datos de la cantidad de taxis que circulan en la ciudad de Pereira.

La llegada de las plataformas digitales ha permitido que muchas personas se aparten de la manera tradicional de compra o de consumo, ya que estas, además de brindar un buen servicio, le dan una gran comodidad a todos los usuarios, los cuales solo tienen que oprimir un botón para solicitar su servicio desde su domicilio. Se debe comprender que hoy por hoy, el éxito no solo depende de contar con las últimas tecnologías, sino también de evolucionar rápidamente y adaptarse a los cambios que estas traen. La innovación es la chispa vital de todo el cambio, mejora el proceso humano. Esto nos ha permitido como seres humanos trascender en distintas épocas y no lo va a dejar de ser (Kelso, 2018).

La economía colaborativa, modelo innovador de negocio, está revolucionando la forma de prestar un servicio haciendo uso de plataformas web a través del intercambio de bienes y servicios entre personas (Watkins, 2010). Millones de personas alrededor del mundo se ven motivadas a entrar en esta nueva modalidad de mercado por el servicio que estas mismas ofrecen y por las experiencias que se pueden vivir entre personas de igual a igual (Rogers, 2011). El factor diferenciador siempre será una estrategia de mercado más apremiante para el ser humano en su diario vivir (Maslow, 2013). Según estadísticas de la compañía Uber, existe un 
crecimiento arrasador tanto de conductores socios como de usuarios afiliados en más de 50 países quienes utilizan la aplicación (Malone, 2014).

Al igual, existen más de 37 millones de reservas en 192 países por aquellos turistas quienes más que pasar una noche, buscan vivir una experiencia diferente al aceptar la oferta de Airbnb. En Colombia, Airbnb empezó a operar en el 2013 en el territorio nacional. Bogotá, Medellín y Cartagena son las ciudades donde existe mayor registro de propiedades en Airbnb y se estima que "ofrecen respectivamente el $9 \%$, el $11 \%$ y el $13 \%$ de la capacidad hotelera de las tres ciudades" (Corrales, 2018). Según estudio dirigido por la Pontificia Universidad Javeriana de Bogotá en un análisis sobre la regulación de Airbnb en Colombia, sustenta que:

Las implicaciones de un sector turístico en crecimiento y una mayor demanda de habitaciones a través de aplicaciones como Airbnb, se evidencia con cifras que estiman que cada noche en Colombia en promedio alrededor [sic] de 12.000 turistas utilizan la plataforma para hospedarse. (Corrales, 2018)

Por todo lo anterior, consideramos pertinente llevar a cabo esta investigación que nos permita ir más a fondo sobre la influencia que existe debido a la creciente tendencia de consumo y utilización de aplicaciones como Uber y Airbnb, que no solo están transformando el sector de transporte y turístico en el país, sino que también están iniciando una serie de discusiones sobre las consecuencias y efectos que generan al mercado tradicional de ambos sectores, a los taxistas y al sector hotelero como competidores.
Cabe resaltar que, teniendo en cuenta el interrogante principal de esta investigación, es necesario analizar qué tan cierto es que estas nuevas plataformas compartidas causan un impacto negativo en los ingresos del sector hotelero y el gremio de taxistas en la ciudad. Además, otra de las razones que lleva a desarrollar el proyecto de investigación radica en el hecho de que existan pocos estudios llevados a cabo sobre el tema en la región. En otras palabras, es necesario realizar estudios que profundicen más sobre el tema, siendo muy conscientes de las nuevas ofertas tecnológicas que existen hoy en el mercado, el nuevo pensamiento económico, social, cultural y político de intercambio de economía, el cual se ha convertido en preferencia a nivel global. A través de la implementación del estudio que se planea llevar a cabo, se podrían llenar algunos espacios por falta de conocimiento sobre el tema, que aún se desconocen o han sido poco investigados.

De esta manera, se tendrían evidencias suficientes para generalizar los resultados a principios más amplios. Por otra parte, es bueno mencionar que serviría para apoyar, comentar o desarrollar una teoría y cómo estudiar más a fondo este fenómeno. Es por ello por lo que la presente investigación pretende recolectar datos sobre el comportamiento de este fenómeno en la ciudad de Pereira y el impacto que genera en la economía del sector hotelero y taxistas.

\section{Materiales y métodos}

Para realizar esta investigación, fue necesario hacer un trabajo de campo para 
ello, los investigadores salieron a hacer encuestas a taxistas y administradores hoteleros, donde se obtuvieron respuestas pertinentes y no se presentaron inconvenientes con los encuestados.

En primer lugar, se encuestaron 92 taxistas donde se evidencia que la mayoría de ellos son de género masculino y oscilan entre los 20 y 80 años; la mayoría de ellos trabaja más de 12 horas diarias. Luego, para el sector hotelero, se realizaron 117 encuestas, igualmente con preguntas muy puntuales y con una aceptación muy positiva de los encuestados.

El método que se utilizó para la investigación es totalmente cuantitativo, para lo cual se usó la encuesta como instrumento de recolección de datos. Para la creación del instrumento, fue necesario hacer una serie de reuniones entre los investigadores para concretar una formulación de preguntas muy claras, para obtener respuestas pertinentes $y$, de esta manera, plasmarlas en una herramienta sistematizada y obtener resultados estadísticos que puedan ser expuestos con mucha claridad. A la hora de la recolección de datos, se destaca la disponibilidad de los encuestados, quienes fueron muy amables y generosos al momento de suministrar sus respuestas. La recolección de la información de los taxistas se pudo hacer en un día completo, mientras que para los hoteles fue necesaria una semana.

\section{Población y muestra}

Taxistas y hoteles: para el cálculo de la

muestra del gremio de los taxistas, se tomaron como población las bases de datos de cinco empresas de taxis que en conjunto reúnen un total de 2687 taxistas. De acuerdo con lo anterior, se calculó la muestra mediante la fórmula de poblaciones finitas, que arrojó un resultado de 92 personas, a las cuales se les aplicará el instrumento diseñado para la recolección de la información.

Para el cálculo de la muestra del gremio hotelero, se tomó como población la base de datos obtenidos de la Cámara de Comercio de Pereira, la cual mostró un total de 180 hoteles registrados. De acuerdo con lo anterior, se calculó la muestra mediante la fórmula de poblaciones finitas, que arrojó un resultado de 117 hoteles, a los cuales se les aplicará el instrumento diseñado para la recolección de la información.

\section{Resultados}

La mayoría de los taxistas encuestados coincidieron en que las plataformas son utilizadas ilegalmente. Además, con base en la pregunta sobre si estarían dispuestos a trabajar en estas plataformas en caso de quedarse sin empleo, las respuestas en un amplio porcentaje fueron un "no" rotundo.

Por otra parte, en el sector hotelero se realizaron 117 encuestas, donde la pregunta más importante fue si conocían alternativas de alojamiento en la ciudad: se obtuvieron respuestas muy diversas, y entre los que respondieron con un sí, muchos coincidieron en que las alternativas eran tales como casas, fincas, hoteles y otros. Además, otra de las preguntas importantes de la encuesta consistió en que si estarían dispuestos a registrar- 
se en una de esas plataformas si tuviera una parte de la casa libre, donde hubo un mayor porcentaje que dijo no.

\section{Conclusiones}

- Los taxistas en Pereira y en toda Colombia no comparten la idea de la economía colaborativa, piensan que es ilegal y persiguen a quienes forman parte de ella.

- Las plataformas digitales han debilitado un poco al gremio de los taxistas y el sector hotelero, pues sustentan que han perdido clientes; por tanto, sus ingresos igualmente.

- Tanto taxistas como personas que trabajan para el sector hotelero, comparten argumentos en decir que las plataformas son ilegales y que no se arriesgarían a trabajar en estas plataformas.

- La mayoría de los taxistas son personas que superan los 35 años de edad, solo un porcentaje muy bajo está entre 20 y 35 años.

\section{Referencias}

1. Botsman, M. (2010). Lo que es mío es tuyo. New York.

2. Corrales, M. P. (2018). ¿Hay cama para tantagente?: unanálisis sobrela regulación de Airbnb en Colombia. Universidad Pontificia Javeriana, Bogotá. https://docplayer. es/140600607-Facultad-de-ciencias-juridicas-hay-cama-para-tanta-gente-un-analisis-sobre-la-regulacion-de-airbnb-en-colombia-autor-martha-patricia-corrales. html

3. DANE. (2017). Reporte: Mercado laboral.
4. Devojeda, K. V. (2013). Accesibilidad basada en los modelos de negocio para los mercados Peer-to-peer. European Commission Business Innovation.

5. Domínguez, S. W. (2003). Creating networks for survival and mobility: Social capital among African American and Latin American low-income mothers. Social Problems, 50(1): 111-135.

6. Durán-Sánchez, A., Álvarez-García, J., La Cruz del, M. d., \& Maldonado-Erazo, C. P. (Sep-Dec de 2016). Economía colaborativa: análisis de la producción científica en revistas académicas. Revista de Gestão e Secretariado; São Paulo, 1-20.

7. Edelman, B. L. (2014). La discriminación digital de Airbnb. Massachussets: Escuela de Negocios de Harvard.

8. Friedman, T. (2013). Bienvenidos a la economía compartida.

9. Hahn, R. (2017). Competencia desleal - Transporte ilegal.

10. Holguín, C. A. (2016). ¿Funcionará la economía colaborativa en Colombia?

11. Kuhndt, M., \& Wagner, T. (2013). Why sharing and swapping instead of owning? How the sharing economy becomes a mass market? Forum Nachhaltig Wirtschaften, accessed on 20/06/2014 via nachaltigwirtschaften.de.

12. Malone, T. R. (2012). La promesa de la economía compartida dentro de comunidades en desventaja. Estado de Michigan: Universidad de Michigan.

13. Navarro-Lozano, H. A. (2004). Using matching, instrumental variables and control functions to estimate economic choice models. Review of Economics and Statistics, 86.

14. Pérez, M. A. (2016). Revista Dinero.

15. Stone, B. (2012). My life as a task rabbit. http://tinyurl.com/9rmptpg. 